Classification

Physics Abstracts

$06.70-43.85$

\title{
Capteur de position à ondes élastiques guidées dans un fil magnétostrictif
}

\author{
E. Dieulesaint, A. Billmann, D. Royer et R. Alkama \\ Université Pierre et Marie Curie, Laboratoire d'Acoustoélectricité, 10, rue Vauquelin, 75231 Paris Cedex 05, France
}

(Reçu le 29 novembre 1984, accepté le 1 er mars 1985)

\begin{abstract}
Résumé. - La position d'un élément mobile est déterminée par la mesure du temps de propagation d'une impulsion élastique. Les expériences décrites, dans lesquelles l'impulsion, engendrée et détectée par effet magnétostrictif, est guidée par une tige de nickel de longueur supérieure à quatre mètres, montrent que cette technique se prête à la réalisation de capteurs de très grande course.
\end{abstract}

\begin{abstract}
The position of a mobile part is determined by the measurement of the propagation time of an acoustic pulse. In the experiments described here the acoustic pulse, generated and detected by magnetostrictive effect is guided by a rod of nickel more than 4 meters long. They show that this technique is appropriate for long range sensors.
\end{abstract}

\section{Introduction.}

Les qualités : sûreté de fonctionnement, précision des machines asservies expliquent le développement de l'automatique. La commande automatique des systèmes exige la connaissance, à chaque instant, de l'état du système par rapport à une référence, c'est-à-dire la saisie en temps réel de grandeurs physiques et en particulier la mesure de la position de pièces en mouvement. Les mesures de position qui doivent être effectuées sans contact mécanique reposent sur des principes différents suivant la course de la pièce mobile, la précision requise, l'environnement. Par exemple, la pièce mobile peut modifier un champ magnétique. Si la course est petite, au plus égale au centimètre, l'élément mobile se déplace dans le champ magnétique fixe, la perturbation du champ magnétique modifie l'inductance d'une bobine dont la variation fournit le déplacement. Si la course est grande, de l'ordre de plusieurs mètres, la pièce "sort" de la région source du champ magnétique sensible à sa position. Il est alors préférable de recourir à une technique du genre Radar (d'autres techniques assurant plus longtemps le contact permanent source-objet et basées sur l'emploi de faisceaux lumineux sont aussi possibles) consistant à envoyer vers l'objet en mouvement une impulsion et à mesurer le temps de propagation aller et retour ou simplement aller si l'objet est coopératif (c'est évidemment le cas ici), c'est-à-dire prévient de

Etude soutenue par le Ministère de la Recherche et de lá Technologie (comité capteurs). l'arrivée de l'impulsion. Cette méthode est difficile à utiliser avec des ondes électromagnétiques, étant donné leur vitesse de propagation $\left(V=3 \times 10^{8} \mathrm{~m} / \mathrm{s}\right)$ et donc les intervalles de temps à mesurer $\left(10^{-2} \mu\right.$ s pour un parcours de $3 \mathrm{~m}$ ). Elle est au contraire facile à exploiter avec des ondes acoustiques dont la vitesse est de l'ordre de quelques milliers de mètres par seconde $(500 \mu$ s pour $3 \mathrm{~m}$ et $V=6000 \mathrm{~m} / \mathrm{s})$. Cependant une onde acoustique se propageant dans l'air est, en général, soumise à des perturbations importantes : sa direction est modifiée si l'indice de l'air change sous l'effet d'une variation de température (effet mirage). Pour soustraire l'onde à ces modifications directes de l'air ambiant, il convient de la guider. L'objet de cet article est de montrer qu'en astreignant un train d'ondes à se propager dans un fil, la méthode se prête à la réalisation de capteurs de course très grande $(>10 \mathrm{~m})$. Les expériences décrites se rapportent à un capteur de course supérieure à $4 \mathrm{~m}$.

\section{Principe.}

Le principe est illustré par le schéma de la figure 1 . A un instant origine, une impulsion électrique est appliquée à un transducteur qui engendre une contrainte mécanique dans le barreau. Au même instant, cette impulsion déclenche une horloge de période $T$. La contrainte se propage à une vitesse constante connue $V$. A son arrivée, au temps $t$, au droit de l'index dont on veut mesurer la position, elle induit, dans un détecteur solidaire de l'index, un signal électrique qui arrête le comptage des tops d'horloge (à la valeur $N$ ) 


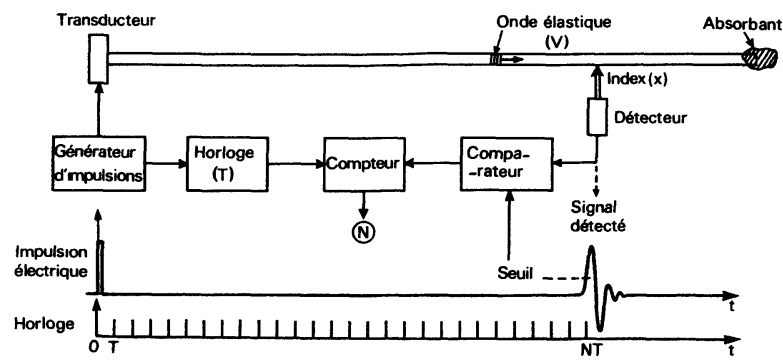

Fig. 1. - L'onde élastique se propage à la vitesse constante $V$. En passant devant l'index elle induit une tension électrique qui arrête le comptage des tops d'horloge (à la valeur $N$ ). L'abscisse de l'index est $x=V N T$.

[The elastic wave propagates at the constant velocity $V$. It passes in front of the pointer inducing an electric voltage which stops the counting of the clock beeps at a number $N$. The abscissa of the pointer is $x=V N T$.]

dès que son amplitude atteint un seuil choisi. L'abscisse de l'index se déduit de la mesure de $t=N T$ :

$$
x=V N T .
$$

A priori, ce principe peut être mis en ouvre de différentes façons : l'impulsion élastique, avec ou sans fréquence porteuse, peut être excitée par effet piézoélectrique ou magnétostrictif. Après s'être propagée au coeur du barreau ou à sa surface, elle peut être détectée par effet piézoélectrique [1], optique [2] ou magnétostrictif [3-5] suivant les propriétés du matériau qui la guide.

Avant de décrire la mise en oeuvre que nous avons adoptée ici, il nous paraît utile de dire quelques mots de la propagation des ondes élastiques dans un barreau et de préciser le mode choisi.

\section{Propagation des ondes élastiques dans un barreau.}

Les vibrations se propageant dans un milieu fini doivent satisfaire à l'équation de propagation et aux conditions aux limites. Comme, ici, la longueur d'un barreau (en fait une tige), plusieurs mètres, est très grande devant son diamètre, quelques millimètres, le barreau peut être considéré comme infini. Le problème de la propagation des ondes guidées se traite alors complètement; résumons les principaux résultats [6]. Trois types de mouvements : compression, torsion, flexion peuvent se propager. A chaque type de mouvement correspond un ensemble de modes dont les vitesses de propagation dépendent du rapport $a / \lambda$, $a$ étant le rayon du cylindre, $\lambda$ la longueur d'onde. Nous nous intéressons aux mouvements de compression parce qu'ils sont relativement plus faciles à exciter; le déplacement de matière est longitudinal, dans l'axe du barreau. La figure 2 représente en fonction de ce rapport $a / \lambda$ la variation de la vitesse $V$ des trois premiers modes. Ces courbes tracées par Davies [7] sont valables pour les matériaux usuels, ayant un

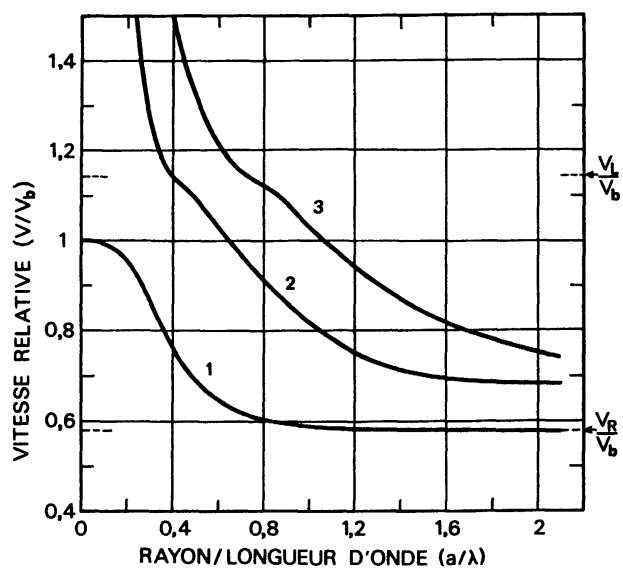

Fig. 2. - Variation de la vitesse de propagation $\left(V / V_{b}\right)$ en fonction du rapport $a / \lambda$ (rayon/longueur d'onde) pour les trois premiers modes de compression dans un barreau de coefficient de Poisson $\sigma=0,29$. D'après R. M. Davies [7].

[Variation of the propagation velocity $\left(V / V_{\mathrm{b}}\right)$ versus the ratio radius/wavelength $(a / \lambda)$ for the first three compressional modes in a bar having a Poisson coefficient $\sigma=0.29$. From R. M. Davies [7].]

coefficient de Poisson voisin de 0,3 (celui du nickel employé ici est 0,31 ). Commentons celle du premier mode. Pour les grandes longueurs d'ondes $(\lambda \gg a)$, c'est-à-dire à basse fréquence, les mouvements latéraux n'interviennent pas; la vitesse est déterminée par le module d'Young $E: V=V_{\mathrm{b}} \equiv \sqrt{E / \rho}, \rho$ étant la masse volumique. Pour les longueurs d'ondes petites devant le rayon, c'est-à-dire à haute fréquence, le mouvement se concentre près de la surface et la vitesse $V$ se rapproche de la vitesse $V_{R}$ des ondes de surface (ondes de Rayleigh).

Dans le cas de matériaux piézoélectriques ou magnétostrictifs dans lesquels un champ électrique ou un champ magnétique est, en général, associé à une déformation, il faut tenir compte du couplage électromécanique. Par ailleurs, les courbes de la figure 2 sont valables pour un état (thermique et mécanique) donné du barreau. L'application d'une tension mécanique, par exemple, entraîne une modification de la vitesse [8]. Cependant la vitesse reste constante, pour une fréquence donnée, si l'état du barreau, homogène, n'est pas modifié.

\section{Expériences.}

Le principe a été appliqué en engendrant et en détectant l'impulsion élastique par effet magnétostrictif à l'aide de deux bobines. Dans une première expérience [9], le fil de nickel avait une longueur de $2 \mathrm{~m}$ et un diamètre de $2 \mathrm{~mm}$. La bobine émettrice était fixe et la bobine réceptrice mobile. L'expérience décrite ici a été effectuée avec un fil de même diamètre $2 \mathrm{~mm}$, mais de longueur $4,3 \mathrm{~m}$. Le fil dont les extrémités sont filetées est tendu entre deux supports (Fig. 3). La 


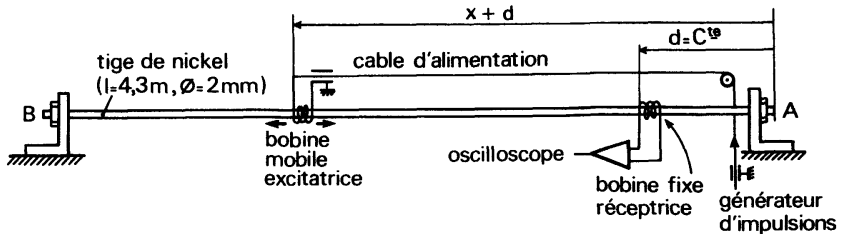

Fig. 3. - La tige de nickel est tendue entre deux contreforts. La bobine mobile excitatrice coulisse librement le long du fil. La bobine réceptrice est fixe.

[The nickel rod is thightened between two supports. The mobile coil slides along the rod. The detecting coil is fixed.]

bobine mobile portant l'index dont on veut mesurer l'abscisse est émettrice : elle reçoit périodiquement des impulsions de courant qui engendrent une variation de l'induction magnétique dans le fil de nickel et donc une déformation élastique donnant naissance à deux ondes se propageant l'une vers la droite, l'autre vers la gauche.

L'induction qui accompagne chaque onde élastique au cours de sa propagation crée une tension aux bornes de la bobine réceptrice fixe. Le diamètre extérieur et la longueur de chaque bobine sont $20 \mathrm{~mm}$ et $10 \mathrm{~mm}$. Chaque bobine est aussi parcourue par un courant d'intensité constante produisant un champ magnétique continu polarisant le fil de nickel. La bobine émettrice est constituée d'un seul enroulement (220 spires de fil $\varnothing=0,45 \mathrm{~mm})$. La bobine réceptrice comprend deux enroulements. L'enroulement détecteur a 2000 spires de diamètre $0,12 \mathrm{~mm}$. L'enroulement de polarisation 4000 spires du même fil.

La photographie 4 montre le signal reçu (amplifié 15 fois) par la bobine réceptrice lorsqu'elle est placée à une distance $d=41 \mathrm{~cm}$ d'une extrémité du fil et séparée de la bobine mobile d'une distance $x$ égale à a) $66 \mathrm{~cm}$, b) $312 \mathrm{~cm}$. La durée de l'impulsion excitatrice

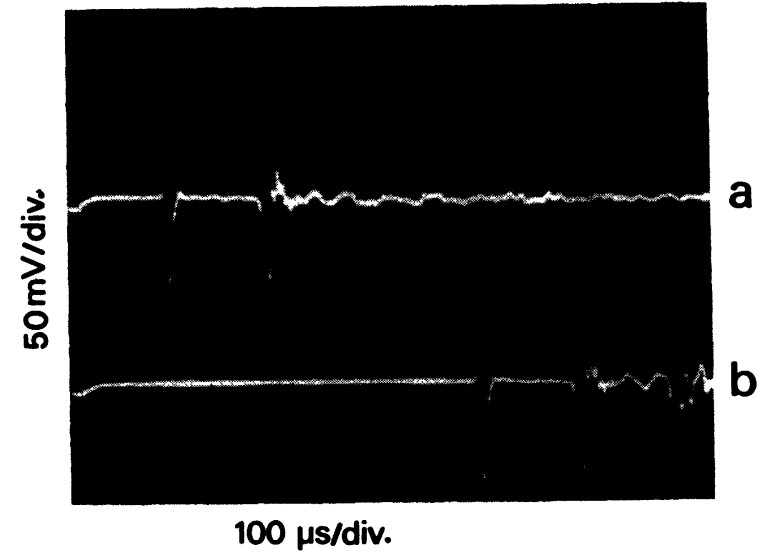

Fig. 4. - Signal détecté aux bornes de la bobine fixe séparée de a) $66 \mathrm{~cm}$, b) $312 \mathrm{~cm}$ de la bobine excitatrice.

[Signal detected by the fixed coil placed at a) $66 \mathrm{~cm}$, b) $312 \mathrm{~cm}$ from the exciting coil.] de courant $(0,1 \mathrm{~A})$ traversant la bobine mobile est $8 \mu \mathrm{s}$. L'intensité du courant de polarisation est environ 0,5 A dans l'enroulement mobile et 0,03 A dans l'enroulement fixe.

L'examen des signaux a et b montrent que le rapport signal/bruit est grand ( $>50 \mathrm{~dB}$ ) et que le parcours de $312 \mathrm{~cm}$ ne donne pas lieu à atténuation.

La deuxième impulsion des signaux $\mathrm{a}$ et $\mathrm{b}$ provient de la partie de l'onde qui après avoir traversé la bobine fixe se réfléchit à l'extrémité A de la ligne. Son retard (ici $162 \mu \mathrm{s}$ ) correspond au parcours supplémentaire $2 d=82 \mathrm{~cm}$.

La troisième impulsion du signal $\mathrm{b}$ provient de la réflexion sur l'extrémité $\mathrm{B}$ de l'onde émise par la bobine mobile alors séparée de cette extrémité de $77 \mathrm{~cm}$ (retard $318 \mu \mathrm{s}$ ).

En pratique, la présence des deuxième et troisième impulsions n'a pas d'effet sur la mesure de $x$, puisque l'horloge est normalement arrêtée par le premier pic dès qu'il atteint un seuil fixé. D'ailleurs, le niveau de ces impulsions-échos pourrait être très sensiblement diminué si la précaution était prise de disposer un matériau absorbant à l'extrémité de la ligne ou de modifier la forme de cette extrémité. Cependant, il pourrait être utile de conserver au moins l'un de ces échos, par exemple le deuxième, dans le cas où il serait nécessaire de corriger un effet produit par une modification de l'environnement (variation de température).

La vitesse de propagation de l'impulsion élastique dans la tige de nickel, déduite de ces mesures, est $4870 \mathrm{~m} / \mathrm{s}$. Compte tenu de la longueur d'onde $\lambda \simeq 5 \mathrm{~cm}$, le point représentatif sur la courbe de dispersion du premier mode de la figure 2 se place tout près de l'origine $(a / \lambda=0,02)$.

Les deux traces sur la photographie 5 représentent les signaux détectés en deux points séparés de $10 \mathrm{~mm}$. Avec un seuil de quelques dizaines de $\mathrm{mV}$, la résolution est de l'ordre de $0,2 \mathrm{~mm}$.

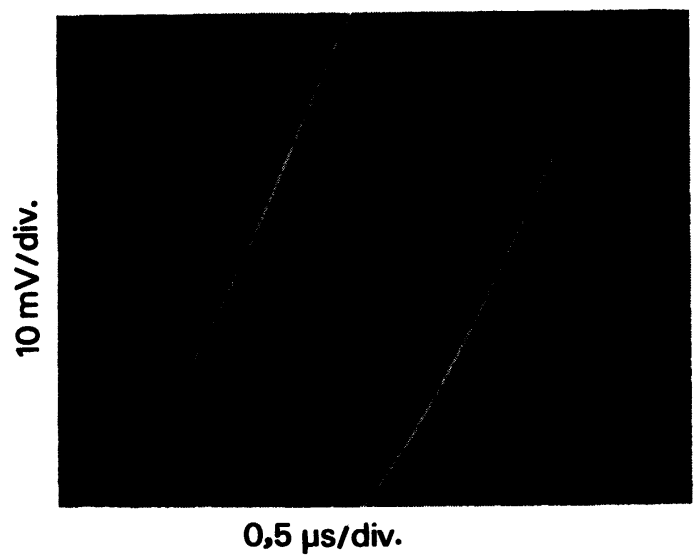

Fig. 5. - Front de montée du signal détecté en deux points distants de $10 \mathrm{~mm}$.

[Rise fronts of the signal detected at two points $10 \mathrm{~mm}$ apart.] 


\section{Conclusion.}

Etant donné l'amplitude des signaux détectés, la variation très faible de cette amplitude avec la distance, ces expériences montrent que la méthode - consistant à exciter à l'aide d'une impulsion de courant une onde élastique dans une tige de nickel et à déduire de son temps de propagation, la position d'un index porté par une bobine captant le champ magnétique associé à la déformation - est applicable à la réalisation de capteurs à course se comptant en mètres et à résolution de quelques points par millimètre. La technique peut être améliorée : les dimensions des bobines, la forme de l'impulsion excitatrice ne sont pas optimales ici.
D'autre part, les effets des variations de température et leur compensation éventuelle sont à étudier. Cependant, on peut déjà penser à des applications; par exemple, en disposant la bobine mobile sur un flotteur, le dispositif est utilisable pour mesurer le niveau d'un liquide dans un réservoir.

\section{Remerciements.}

Nous remercions M. Duchaussoy de la DRET et M. Louvel de la Société Elf-Aquitaine de leurs conseils et de l'attention qu'ils portent à ce travail.

\section{Bibliographie}

[1] Dieulesaint, E., Royer, D. and Ballegeer, J. C., Electron. Lett. 12 (1976) 586.

[2] Dieulesaint, E., Royer, D. and Sitbon, G., IEEE Ultrason. Symp. Proc. (1980) 480.

[3] Worthington, T. K., Thompson, D. A., IEEE Trans. Magn. MAG-16 (1980) 693.

[4] Chamuel, J. R., Ultrason. Internat. Proc. (Brighton 1981) 342.

[5] Van Den Berg, K. G.,.J. Phys. E : Sci. Instrum. 15 (1982) 325 .
[6] AChenbach, J. D., Wave Propagation in Elastic Solids (North-Holland, Amsterdam) 1975, p. 236.

[7] Davies, R. M., Phil. Trans. R. Soc. A 240 (1948) 375.

[8] Hashimoto, K. Y., Yoshida, M., Yamaguchi, M. and Kogo, H., Electron. Lett. 16 (1980) 425.

[9] Billmann, A., Dieulesaint, E., Royer, D. et Alkama, R., Capteurs 84 CIAME, Recueil des communications, 199 (Paris 1984). 